\title{
Qualitative analysis of the deposit of collagen in bladder suture of rats treated with tacrolimus combined with mycophenolate-mofetil
}

\author{
Gustavo Marquesine Paul, Renato Tambara Filho, João Carlos Domingues Repka \\ Department of Clinical Surgery. Health Sciences Sector of the Federal University of Parana (UFPR), Curi- \\ tiba, PR, Brazil
}

\section{ABSTRACT}

Purpose: To evaluate the synthesis of type I (mature) and type III (immature) collagen in bladder suture of rats treated with a combination of tacrolimus and mycophenolate mofetil for 15 days.

Materials and Methods: Thirty rats were divided into 3 groups: the sham, control and experimental groups. All the animals underwent laparotomy, cystotomy and bladder suture in two planes with surgical PDS 5-0 thread. The sham group did not receive treatment. The control group received saline solution, and the experimental group received $0.1 \mathrm{mg} / \mathrm{kg} /$ day of tacrolimus with $20 \mathrm{mg} / \mathrm{kg} /$ day of mycophenolate mofetil, for 15 days. From then on, the tacrolimus was dosed. The surgical specimens of the bladder suture area were processed so that the total type I and type III collagen could be measured by the picrosirius red technique.

Results: There was a predominance of type I collagen production in the sham and control groups compared to the experimental group, in which type III collagen was predominant. The production of total collagen did not change.

Conclusion: The association of tacrolimus and mycophenolate mofetil in animals qualitatively changes the production of collagen after 15 days with a predominance of type III collagen.

\section{ARTICLE INFO}

\section{Key words:}

Wound Healing; Immunosuppression; Collagen; Urothelium

Int Braz J Urol. 2014; 40: 257-65

Submitted for publication: March 25, 2013

\section{Accepted after revision:}

January 21, 2014

\section{INTRODUCTION}

Urological complications increase the morbidity and mortality of kidney transplantation by increasing the length of hospital stays and the need for re-surgery (1). The incidence of urological complications after kidney transplantation ranges from $2.5 \%$ to $14.7 \%$ (2). The main urological complications in kidney transplants are ureteral strictures and urinary fistulas (3). Most urinary fistulas appear early, within the first 90 days postoperatively (4).
After tissue damage, the process of restoring the tissue through a series of biochemical and physiological cellular processes begins (5). Collagen has a special feature. It is the main protein of connective tissue, responsible for the mechanical strength and resistance of the scar tissue. Regardless of the injured tissue, collagen is the most important component in tissue repair (6). Type I (mature) collagen is the most frequent. It is synthesized by fibroblasts and predominant in bones and tendons. Type III (immature) collagen is most commonly found in soft tissues, such 
as blood vessels, dermis and fascia. The physical characteristic that best distinguishes type I collagen from type III is the interlacing of their fibers. The fibers of type I collagen are more intertwined and compacted than those of type III collagen, which has little interlacing, which results in lower tensile strength for scar tissue. The strength of a suture can be evaluated by the ratio of immature and mature collagen (7). These qualitative characteristics of deposited collagen are important for the structural support of an anastomosis. The maximum deposition of collagen in healing tissue is found on the fifteenth day (8). Every organ has a varying capacity for tissue repairs. The bladder has different characteristics when compared to gastrointestinal tract regeneration (9).

The picrosirius red staining technique stands out due to its greater selectivity for conjunctive tissue (10). This staining is specific for collagen, since there are no strong stains on the glycoprotein fibers (11). The less interwoven collagen fibers, representing type III collagen, are represented in green. The more interwoven fibers, aligned and with strong staining, representing type I collagen are orange-red (12). The calculation of the percentage of fibers, classified as type I or type III according to their color, enables a qualitative assessment of collagen fibers (13).

Among the various factors that may affect wound healing, immunosuppression is an important factor that hinders the healing process (14). There are various immunosuppressive regimens, and these drugs are based on calcineurin inhibitors, with cyclosporine and tacrolimus being the most commonly used. The most studied adjuvant drugs are mycophenolate mofetil and sirolimus. A combination of tacrolimus with mycophenolate mofetil is more commonly used nowadays (15).

This experimental study with rats aimed to verify the effect of the combination of Tacrolimus and Mycophenolate Mofetil on the synthesis of types I and III collagen in bladder wound healing.

\section{MATERIALS AND METHODS}

\section{Animals and groups}

We observed the ethical principles in animal experimentation established by the Brazilian
School of Animal Experimentation (COBEA). Thirty Wistar rats, aged 120-140 days and weighing $265.34 \pm 23.73$ grams were used. They were divided into 3 groups of 10: the sham, control and experimental groups.

\section{Surgical technique}

The rats were weighed and submitted to inhalation of halothane sedation and anesthesia by intramuscular injection of ketamine and xylazine hydrochloride. A four-centimeter longitudinal midline incision was made in the following sequence: skin, subcutaneous tissue, rectus abdominis muscles and peritoneum. The isolated urinary bladder of the animal was subjected to a three-centimeter longitudinal cystotomy in the anterior bladder wall. The defect was closed with 5-0 polydioxanone suture in two planes. The closure of the abdominal cavity was done with Polyglactin 910 (3-0) thread, and the skin closure with simple colorless nylon (3-0) thread.

The animals in the sham group received no specific treatment after the surgery procedure. The animals in the control group were subjected to the same conditions of sedation and received daily subcutaneous injections and oral saline solution in volume proportional to their weight. The rats in the experimental group received daily treatment with tacrolimus and mycophenolate mofetil. The tacrolimus was administered subcutaneously on a daily basis at a dose of $0.1 \mathrm{mg} / \mathrm{kg} /$ day for 15 days and mycophenolate mofetil daily dose of $20 \mathrm{mg} /$ $\mathrm{kg} /$ day for fifteen days, administered orally (4).

By the fifteenth day of evolution, all the rats were sedated and underwent cardiac puncture for blood collection. The blood samples were sent to the laboratory in order to perform a clinical analysis of tacrolimus (16). After the death of the animals, samples were collected from the bladder wall. The sample was then sent for the determination of total type I and type III collagen tissue by the histological technique of picrosirius red (17).

\section{Optical microscopy}

We assessed the area, density and the percentage of type I and III collagen. For identification of type III and type I collagen, the sections were analyzed by an Olympus ${ }^{\circledR}$ brand optical 
microscope with 400 times magnification under polarized light. The images were captured by an optical system, frozen and scanned. This was performed by image analysis application using Image-Pro Plus version 4.5 for Windows (RGB). This program identifies the type of collagen-based colors. Red, yellow and orange correspond to type I collagen (mature), whereas green is equal to type III collagen (immature). Three fields were evaluated (upper, middle and lower), perpendicular to the suture bladder. The result was expressed as a percentage area.

A descriptive analysis of the data was applied to graphs and charts. The Student $t$ and ANOVA parametric tests were used with the GraphPad application and a significance level of less than $5 \%(\mathrm{p}<0.05)$ was adopted.
In histopathological reviews concerning the histometric assessment of the areas of total collagen, when the values of the areas occupied by the total collagen of the groups were compared, there was no statistical difference, as shown in Figure-1. The control group had a mean of $22,728,734.89 \pm 8,535,056.23 \mu^{2}$, the sham group 20,280,575.18 $\pm 6,637,851.96 \mu^{2}$, and the experimental group 20,467,537.37 \pm $8,946,377.93 \mu^{2}$ total collagen. There was no significant difference between the groups ( $p=$ 0.7558).

The histology assessment of the areas of type I collagen (Figure-2) showed that in the control group the average mature collagen detected was $95.94 \pm 2.28 \%$, in the sham group $94.76 \pm$ $4.05 \%$, and in the experimental group, $4.95 \pm$

Figure-1 - Means and standard deviations areas of total collagen.

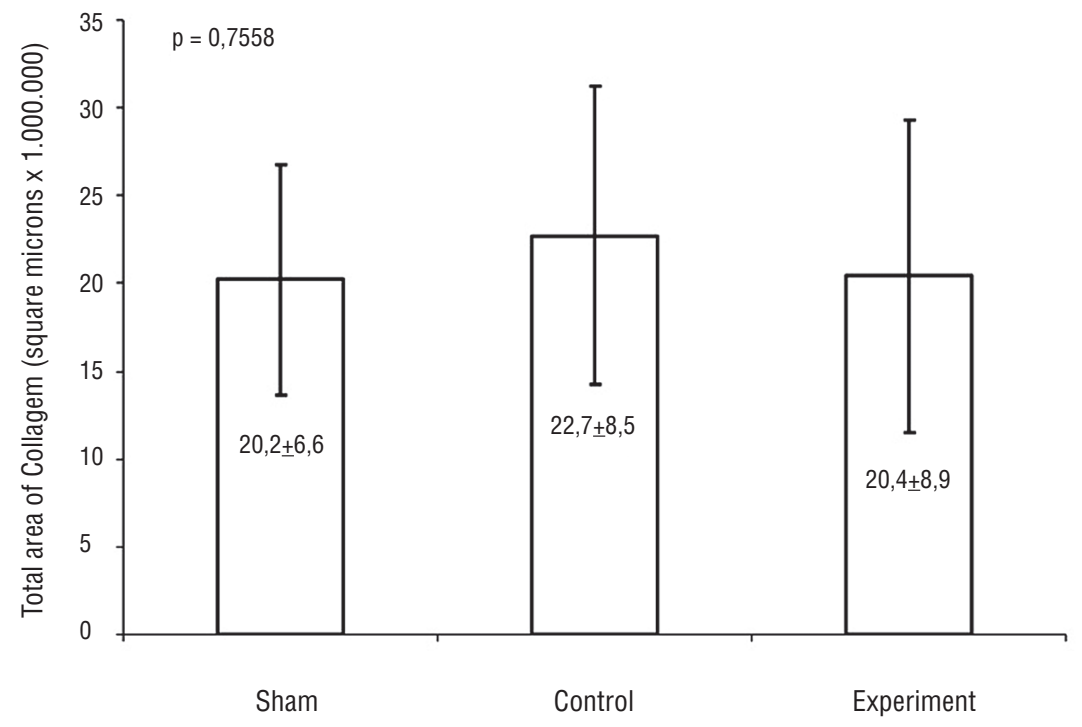

\section{RESULTS}

Regarding the dosage of tacrolimus it was observed that no serum levels of the drug were detected in the sham and control groups. In the experimental group an average of $11.3 \pm 2.07 \mathrm{ng} /$ $\mathrm{mL}$ of tacrolimus was detected.
$3.97 \%$ of mature collagen (type I) in square microns $x$ 1.000.000. There was no statistically significant difference between the control and sham groups $(p=0.4362)$. Comparing the sham and experimental groups, there was a statistically significant difference $(\mathrm{p}<0.0001)$, and also between the control and experimental groups $(\mathrm{p}<0.0001)$. 
Figure-2 - Mean and standard deviations densities of type I and type III collagen (percentage area).

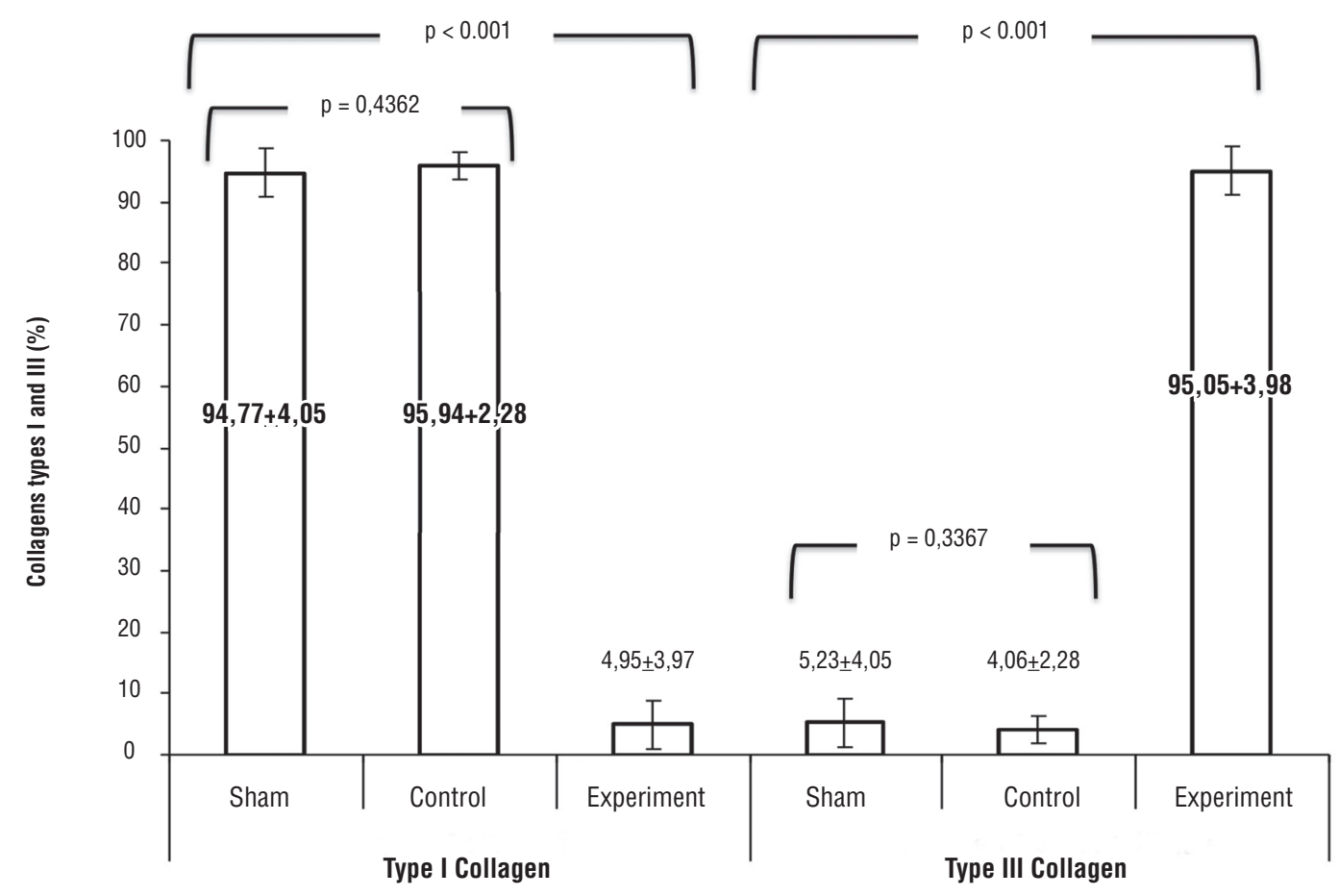

Concerning the histological evaluation of areas of type III collagen (Figure-2), in the control group the average immature collagen detected was $4.06 \pm 2.28 \%$, in the sham group, $5.23 \pm 4.05 \%$ and in the experimental group, $95.04 \pm 3.97 \%$, in square microns $x 1,000,000$. Between the control and sham groups, there was no statistically significant difference $(p=0.3307)$. Comparing the sham and experimental groups, there was a statistically significant difference $(p<0.0001)$, as there was between the control and experimental groups $(\mathrm{p}<0.0001)$.

Figure-3 shows the histological sections stained with Sirius red F3BA (40x). On the left picture, shown in red, type I collagen, and on the right picture in blue-green, type III collagen, under polarized light.

\section{DISCUSSION}

The main contribution of this study is that it demonstrates the qualitative change in the synthesis of collagen in a bladder wound in rats sub- jected to pre-defined immunosuppression drugs. There are several studies showing the complications and healing changes in the presence of immunosuppression, but few are prospective and well controlled. There have been many studies of other tissues such as the skin and gut, but little in urothelium (6). In this study, there was significant reduction in the production of type I collagen, using immunosuppression with tacrolimus and mycophenolate mofetil after fifteen days of the experiment. In the present study, the choice of immunosuppression was based on numerous studies that demonstrate the advantages of the combination of tacrolimus and mycophenolate mofetil (15). International study protocols (SWTC) see no statistical difference between immunosuppression with tacrolimus and cyclosporine as indices of acute rejection, but there is a trend of longer survival with the use of tacrolimus. The use of mycophenolate mofetil significantly reduces the incidence of rejection when compared with azathioprine (3). Tacrolimus should be monitored to prove the therapeutic concentration of the tacrolimus (16). In this study, 
drugs were only detected in the samples of the experimental group, with all doses falling within the therapeutic range of the drug.

Many authors have reported the deleterious effect of immunosuppression on wound healing and most of these studies do not analyze type I and type III collagen separately. In practice, we observed that in tissue healing, type III collagen can indeed be a precursor of type I collagen as it has a lower quantity of fibers, less intertwined fibers and a lower quantity of local cellularity. Among the articles that studied tissue healing in non-urothelial tissue, one study that stands out is that of Kita et al. (18), who looked at the healing of the small intestine and colon of rats, observed that the tensile strength (bursting pressure of the anasto-

Figure-3 - Histological method of sirius red f3ba (40x).

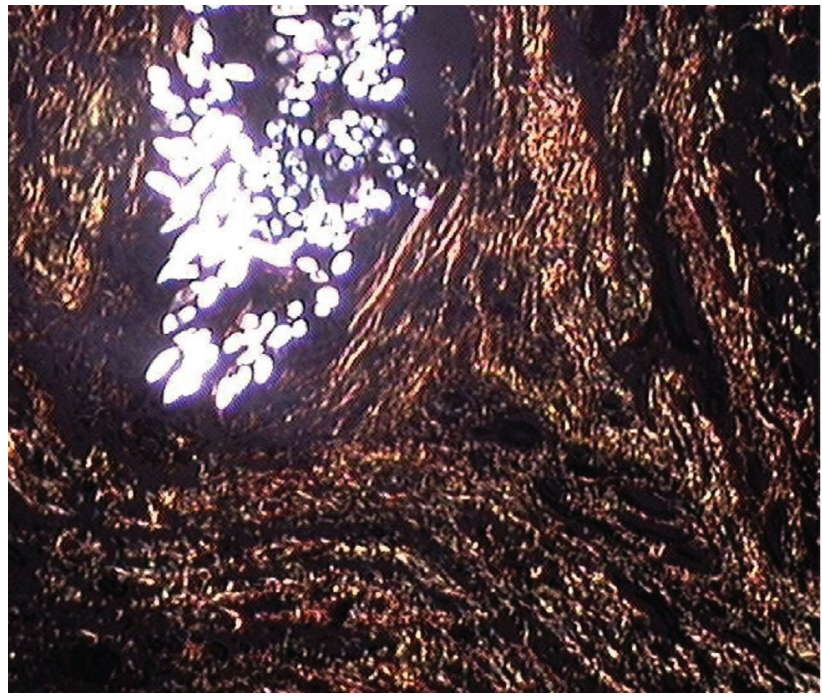

mosis) of colonic anastomoses was less resistant in animals treated with tacrolimus at the end of seven days of treatment with tacrolimus for via intra-peritoneal. Furthermore, Schaffer et al. (19) studied the effects of tacrolimus in the healing of intestinal tissue and dermis and observed that the administration of $2 \mathrm{mg} / \mathrm{kg}$ of tacrolimus led to a reduction in the healing dermis of animals. On the other hand, regarding the study of urothelial tissue, Ekici et al. (20) looked at the effects of immunosuppression with sirolimus in the healing of sutures in the bladder of rats and concluded that sirolimus affects all stages of healing of the bladder, including reducing the number of inflammatory cells, angiogenesis and the proliferation of myofibroblasts, thus delaying the healing process.

Some more recent works are using immunosuppressant drugs such as tacrolimus in the study of the treatment of diseases involving cellular proliferation disorders. Of these articles on cellular biology, one that deserves to be mentioned is that of $\mathrm{Wu}$ et al. (21). Concerning the behavior of keloid fibroblasts activated with tissue growth factors (TGF- $\beta 1$ ), this study concluded that tacrolimus inhibits the growth factor action on the fibroblast in vitro. Inhibiting the proliferation of fibroblasts and their tissue migration, the entire protein synthesis of

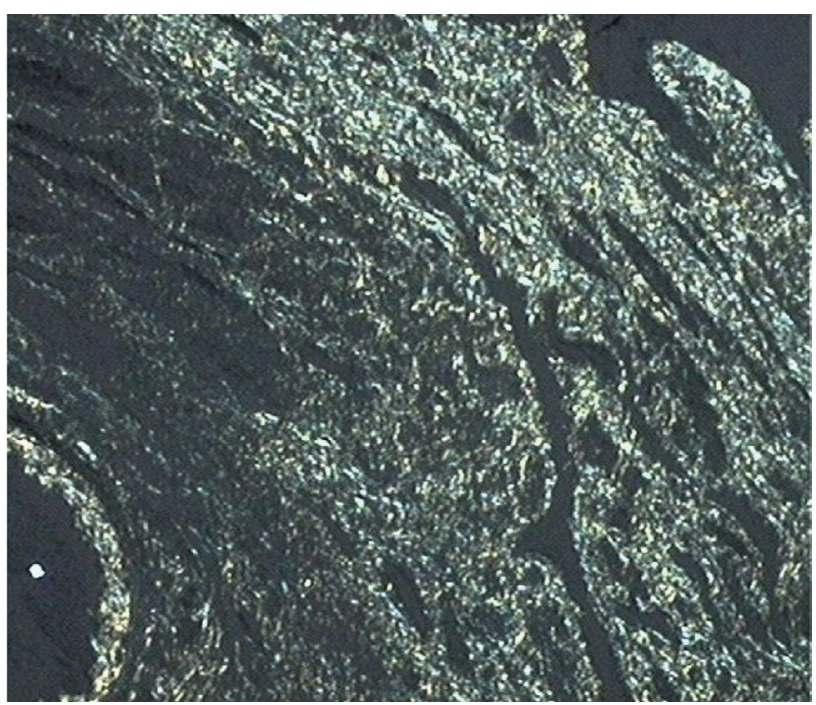

tissue collagen is impaired. Following the same line of research, Nankoong et al. (22) studied the effect of the topical tacrolimus therapy in the healing of cutaneous wounds in the backs of mice. They observed that after 3, 7 and 11 days of healing, there was no significant alteration in the healing of tissue between the groups under study, but the group treated with tacrolimus had slightly reduced levels of expression of mRNA of IL- $1 \alpha$ and TGF- $\beta$.

Even topical therapy with tacrolimus appears to reduce local fibrosis. Ismailoglu et al. 
observed that topical therapy with tacrolimus in the dura-mater of rats submitted to laminectomy reduced the occurrence of local fibrosis. The animals treated after thirty days with tacrolimus had a reduced amount local distribution of fibroblasts and reduced local fibrosis (23). But the most interesting study was certainly that in which Raptis et al. (24) observed that tacrolimus, when employed in healing the colons of rats, after 4 and 8 days of study reduced the occurrence of inflammatory reactions and the presence of local type I collagenase, although it increased the hydroxyproline concentration, neo-angiogenesis and the bursting pressure of anastomosis in the colons of the rats. Finally, Que et al., studying the regeneration of sciatic nerves in rats observed that tacrolimus reduces the formation of scar tissue in the area of the wound. These authors also observed that this reduction is associated with reduced proliferation and the apoptosis of fibroblasts induced by tacrolimus (25).

A joint analysis of our experiment with the literature shows that the immunosuppressive scheme that uses calcineurin inhibitors such as tacrolimus leads to a reduction in the proliferation of fibroblasts and the production of collagen. This reduces the amount of residual scar tissue. However, not all the studies that analyzed the bursting tension of anastomosis in these animals found that the animals treated with the immunosuppression scheme saw worsened bursting tension in their anastomosis, with some even noting increased bursting tension of anastomosis with the use of tacrolimus, in studies with a shorter trial period. More studies correlating the presence of type I collagen, type III collagen and tissue bursting pressure in the urothelium are required.

However, we need to take into account that our study is experimental, conducted in rats and with a short time frame for evaluating the results. We do not know whether these alterations in qualitative production of collagen will be maintained beyond the fifteen days of this study. For the time being, we cannot consider these results directly for clinical practice on human beings, where the scenario tends to be more complex and involves some variables that were not evaluated during the present study.

\section{CONCLUSIONS}

The combination of immunosuppressant tacrolimus and mycophenolate mofetil qualitatively alters collagen synthesis, resulting in increased production of proportional type III collagen after fifteen days of treatment at the doses used in rats.

\section{CONFLICT OF INTEREST}

None declared.

\section{REFERENCES}

1. Mäkisalo H, Eklund B, Salmela K, Isoniemi $H$, Kyllönen $L$, Höckerstedt K, et al.: Urological complications after 2084 consecutive kidney transplantations. Transplant Proc. 1997; 29: 152-3.

2. Humar A, Ramcharan T, Denny R, Gillingham KJ, Payne WD, Matas AJ: Are wound complications after a kidney transplant more common with modern immunosuppression? Transplantation. 2001; 72: 1920-3.

3. Agüera L, Robles JE, Rodríguez-Rubio Fl, Abad Jl, Rosell D, Zudaire JJ, et al.: Prognostic implications of urological complications in the renal transplantation. Rev Med Univ Navarra. 1994; 38: 195-200.

4. Nicoluzzi J, Silveira F, Porto F, Macri M: One hundred pancreas transplants performed in a Brazilian institution. Transplant Proc. 2009; 41: 4270-3.

5. Witte MB, Barbul A: General principles of wound healing. Surg Clin North Am. 1997; 77: 509-28.

6. Thornton FJ, Barbul A: Healing in the gastrointestinal tract. Surg Clin North Am. 1997; 77: 549-73.

7. Koruda MJ, Rolandelli RH: Experimental studies on the healing of colonic anastomoses. J Surg Res. 1990; 48: 504-15.

8. Hendriks T, Mastboom WJ: Healing of experimental intestinal anastomoses. Parameters for repair. Dis Colon Rectum. 1990; 33: 891-901.

9. Edlich RF: The biology of wound repair and infection: a personal odyssey. Ann Emerg Med. 1985; 14: 1018-25.

10. Sweat F, Puchtler H, Rosenthal SI: Sirius red F3ba as a stain for connective tissue. Arch Pathol. 1964; 78: 69-72.

11. Junqueira LC, Bignolas G, Brentani RR: Picrosirius staining plus polarization microscopy, a specific method for collagen detection in tissuesections. Histochem J. 1979; 11: 447-55. 
12. Dayan D, Hiss Y, Hirshberg A, Bubis JJ, Wolman M: Are the polarization colors of picrosirius red-stained collagen determined only by the diameter of thefibers? Histochemistry. 1989; 93: 27-9.

13. Rabau M, Eyal A, Kluger $Y$, Dayan D: Bursting pressure in anastomotic healing in experimentally induced colitis in rats. Dis Colon Rectum. 1998; 41: 468-72.

14. Schäffer MR, Fuchs N, Proksch B, Bongartz M, Beiter T, Becker HD: Tacrolimus impairs wound healing: a possible role of decreased nitric oxide synthesis. Transplantation. 1998; 65: 813-8.

15. Sampaio EL, Pinheiro-Machado PG, Garcia R, Felipe CR, Park SI, Casarini DE, et al.: Mycophenolate mofetil vs. sirolimus in kidney transplant recipients receiving tacrolimusbasedimmunosuppressive regimen. Clin Transplant. 2008; 22: $141-9$.

16. Winkler M, Ringe B, BaumannJ, Loss M, WonigeitK, Pichlmayr $\mathrm{R}$ : Plasma vs whole blood for therapeutic drug monitoring of patients receiving FK 506 forimmunosuppression. Clin Chem. 1994; 40: 2247-53.

17. Junqueira LC, Cossermelli W, Brentani R: Differential staining of collagens type I, II and III by Sirius Red and polarization microscopy. Arch Histol Jpn. 1978; 41: 267-74.

18. Kita J, Ogino Y, Kobayashi E, Fujimura A, Kogure H: Effects of tacrolimus on small and large bowel anastomoses in the rat. Transplant Proc. 1999; 31: 2789.

19. Schäffer M, Fuchs N, Völker J, Schulz T, Kapischke M, Viebahn R: Differential effect of tacrolimus on dermal and intestinal wound healing. J Invest Surg. 2005; 18: 71-9.
20. Ekici $Y$, Emiroglu R, Ozdemir $H$, Aldemir D, Karakayali $H$, Haberal M. Ekici Y, Emiroglu R, Ozdemir H, Aldemir D, Karakayali $\mathrm{H}$, Haberal M: Effect of rapamycin on wound healing: an experimental study. Transplant Proc. 2007; 39: 1201-3.

21. Wu CS, Wu PH, Fang AH, Lan CC: FK506 inhibits the enhancing effects of transforming growth factor (TGF)- $\beta 1$ on collagen expression and TGF- $\beta /$ Smad signalling in keloid fibroblasts: implication for new therapeutic approach. $\mathrm{Br} \mathrm{J}$ Dermatol. 2012; 167: 532-41.

22. Namkoong S, Chung J, Yoo J, Jung M, Gye J, Kim JS, et al.: Topical tacrolimus does not negatively impact acute skin wound healing. Exp Dermatol. 2013; 22: 369-71.

23. Ismailoglu 0, Albayrak B, Gulsen I, Tanriover G, Demir N: Topical application of tacrolimus prevents epidural fibrosis in a rat postlaminectomy model: histopathological and ultrastructural analysis. Turk Neurosurg. 2011; 21: 630-3.

24. Raptis D, Mantzoros I, Pramateftakis MG, Despoudi K, Zaraboukas T, Koliakos G, et al.: The effects of tacrolimus on colonic anastomotic healing in rats. Int J Colorectal Dis. 2012; 27: 299-308.

25. Que J, Cao Q, Sui T, Du S, Kong D, Cao X: Effect of FK506 in reducing scar formation by inducing fibroblast apoptosis after sciatic nerve injury in rats. Cell Death Dis. 2013; 4: e526.

Correspondence address:

Gustavo Marquesine Paul, MD

Rua Portugal, 307, São Francisco 80510-280, Curitiba, PR, Brazil

Telephone: +55 41 3074-7497

E-mail: gustavompaul@gmail.com 


\section{EDITORIAL COMMENT}

Nowadays the association of a calcineurin inhibitor (CNI) with Mycophenolate mofetil (MMF) represents the backbone of solid-organ transplant immunosuppression. Although CNIs [Cyclosporine A (CsA) and Tacrolimus (FK506)] remain the most effective and widely used immunosuppressive agents in organ transplantation, their prolonged use may result in renal toxicity, renal dysfunction and irreversible renal failure characterized by extensive tubulo-interstitial fibrosis. The immunosuppressive effect of CNIs depends on the formation of a complex with their cytoplasmic receptor that inhibits calcineurin and impairs the expression of several cytokine genes that promote T-cell activation such as IL-2, IL-4, INF- $\gamma$ and TNF- $\alpha$ (1). Moreover CNIs induce the expression of TGF- $\beta 1$, which contribute to IL-2 inhibition but it is the main responsible for the development of CNI-associated interstitial fibrosis. TGF- $\beta 1$ is well recognized as the major inducer for tissue fibrosis due to its stimulatory effect on extracellular matrix (ECM) production and inhibitory effect on matrix metalloproteinases. Recently it has been suggested that epithelial-mesenchymal transition (EMT) could play a role in the progression and maintenance of fibrosis in many pathological conditions, including tubulo-interstitial fibrosis (2). EMT is defined as the acquisition by epithelial cells of the phenotypic and functional characteristics of mesenchymal cells, intermediate between fibroblast and smooth muscle cells. These myofibroblasts have the ability to produce and secrete the extracellular matrix components such as collagen I and III, fibronectin and express $\alpha$-smooth muscle actin ( $\alpha$-SMA). It has been shown that long-term exposure to CsA, induces EMT in human proximal tubular cells and that this event is mediated by CsA-induced TGF- $\beta 1$ secretion (2). Moreover it has been observed that Tacrolimus up-regulates the expression of TGF- $\beta$ and Smad2 in renal graft, while MMF has opposite effects (3). In fact it has been reported that MMF can reduce transplant fibrosis in a rat model of chronic rejection possibly by reducing the expression of $\alpha$-SMA, collagen and connective tissue growth factor (CTGF), a matricellular protein with an important role in fibrosis and EMT (3). In accordance with these findings, Jiang et al. showed that MMF treatment prevented the deterioration of renal function and interstitial fibrosis in a renal ischemia-reperfusion injury model (4). In particular MMF significantly reduced the macrophages infiltration and the tissue expression of TGF- $\beta 1$ and MCP- 1 , a diagnostic marker of renal injury (5).

In this scenario $\mathrm{Wu}$ et al. investigated the effects of Tacrolimus in wound healing, especially in a particular pathological process characterized by aberrant fibroblast activity with development of keloids (6). These authors showed that Tacrolimus could inhibit the TGF- $\beta 1$-stimulated cell proliferation, migration and type I collagen production in keloid fibroblasts via Smad-related pathways inhibition (6). A fundamental characteristic of tissue fibrosis is the deregulated deposition of ECM, especially type I and III collagen. The imbalance between matrix metalloproteinases (MMPs) and their specific inhibitors (TIMPs: tissue inhibitors of MMFs) may lead to ECM accumulation and tissue fibrosis. Lan et al. showed that the use of Tacrolimus increased MMPs production and decreased TIMPs, with abrogation of TGF- $\beta 1$-induced type I collagen synthesis (7).

The present study provides new insights into biological effect of Tacrolimus-MMF combination on the collagen synthesis in bladder wound healing. Even if the specific effect of the single immunosuppressive drugs was not addressed separately, for the first time it has been clearly shown a qualitative alteration in collagen synthesis characterized by a switch from type I to type III deposition. Understanding the mechanisms involved in tissue fibrosis may lead to the development of novel strategies for the treatment of CNIs-associated nephrotoxicity with the aim to increase graft survival.

\section{REFERENCES}

1. Halloran PF: Immunosuppressive drugs for kidney transplantation. N Engl J Med. 2004; 351: 2715-29. Erratum in: N Engl J Med. 2005; 352: 1056.

2. Slattery C, Campbell E, McMorrow T, Ryan MP: Cyclosporine A-induced renal fibrosis: a role for epithelial-mesenchymal transition. Am J Pathol. 2005; 167: 395-407. 
3. Luo L, Sun Z, Wu W, Luo G: Mycophenolate mofetil and FK506 have different effects on kidney allograft fibrosis in rats that underwent chronic allograft nephropathy. BMC Nephrol. 2012; 13: 53.

4. Jiang $\mathrm{S}$, Tang $\mathrm{Q}$, Rong $\mathrm{R}$, Tang $\mathrm{L}, \mathrm{Xu} \mathrm{M}$, Lu J, et al.: Mycophenolate mofetil inhibits macrophage infiltration and kidney fibrosis in long-term ischemia-reperfusion injury. Eur J Pharmacol. 2012; 688: 56-61.

5. Lucarelli G, Ditonno P, Bettocchi C, Grandaliano G, Gesualdo L, Selvaggi FP, Battaglia M: Delayed relief of ureteral obstruction is implicated in the long-term development of renal damage and arterial hypertension in patients with unilateral ureteral injury. J Urol. 2013; 189: 960-5.

6. Wu CS, Wu PH, Fang AH, Lan CC: FK506 inhibits the enhancing effects of transforming growth factor (TGF)- $\beta 1$ on collagen expression and TGF- $\beta /$ Smad signalling in keloid fibroblasts: implication for new therapeutic approach. $\mathrm{Br} \mathrm{J}$ Dermatol. 2012; 167: 532-41.
7. Lan CC, Fang AH, Wu PH, Wu CS: Tacrolimus abrogates TGF- $\beta 1$-induced type I collagen production in normal human fibroblasts through suppressing p38MAPK signalling pathway: implications on treatment of chronic atopic dermatitis lesions. J Eur Acad Dermatol Venereol. 2013; 10. [Epub ahead of print]

Giuseppe Lucarelli, MD University of Bari

Department of Emergency and Organ Transplantation Piazza G. Cesare, 11 70124 Bari, Italy E-mail: giuseppe.lucarelli@inwind.it

Pasquale Ditonno, MD University of Bari Department of Urology, Andrology and Kidney Transplantation Unit Piazza G. Cesare, 11 70124 Bari, Italy E-mail: pasquale.ditonno@uniba.it 